\title{
Measurement of Impedance Values of Different Erythrocyte Suspensions
}

\author{
(D) Mehmet ÜYÜKLÜ
}

Bezmialem Vakıf University Faculty of Medicine, Department of Physiology, İstanbul, Turkey

\begin{abstract}
Objective: The study was aimed to determine whether the impedance measurements of erythrocyte suspensions can be used with a different calculation method in determining the degree of erythrocyte aggregation.

Methods: Impedance measurements of different erythrocyte suspensions were recorded in horizontal glass capillaries with a haematocrit value of $40 \%$ with inductance-capacitance-resistance meter after a stop flow generated by the injector pump.

Results: As a result of calculating the impedance values of the samples for which the aggregation in phosphate-buffered saline didn't occur, the impedance values of the samples that aggregation at different grades took place were calculated as in the previous measurements of erythrocyte suspensions time course was significantly decreased for in diluted plasmas and was increased for in the $1 \%$ dextran 500 solution. It is obvious that aggregation index (AI) was calculated, using $\mathrm{Z}$ data exhibited a similar trend for the diluted plasma and plasma with $1 \%$ dextran 500 , with a rank order of Dextran $500>$ whole blood $>1 / 2$ diluted plasma.
\end{abstract}

Conclusion: Although impedance measurements of erythrocyte suspensions don't allow for the calculation of erythrocyte aggregation kinetics, it is thought that they can be used as AI which indicates the degree of erythrocyte aggregation.

Keywords: Electrical measurements, impedance, capacitance, red blood cell aggregation

\section{Introduction}

Aggregation give rise to erythrocyte surfaces parallel to each other and clustering in a special way. Erythrocyte aggregation occurs only in the suspension phase in the presence of a certain size and structure of macromolecules. This clustering does not occur when the same cells suspended in simple salt solutions. Factors affecting red blood cell (RBC) aggregation can be divided into intrinsic and extrinsic factors: (1) Extrinsic factors include levels of plasma proteins [exempli gratia (e.g.), fibrinogen, macroglobulins], hematocrit and shear rate; (2) Intrinsic factors include RBC shape, deformability and membrane surface properties (1-3)

Aggregation can be altered during pathophysiological processes due to modifications of both plasma composition (e.g., in acute phase reactions) and cellular factors (e.g., increased oxidant stress) (3-5) RBC aggregation affects the flow properties of blood, especially at low shear rates, and therefore has the potential to influence blood flow in the circulatory system (6). The factors determining RBC aggregation could be classified in two groups, extracellular and cellular. The extracellular factors are: flow condition, concentration of plasma proteins (fibrinogen, immunoglobulins, albumin etc.), the presence of other macromolecules (e.g. dextrans), pHvalue, osmotic pressure. The cellular factors are: the glycocalyx, determines kinetics, and extent of the contact area between the cells and so the rate and the strength of the aggregation. For example glutaraldehyde treatment of RBC results in intra- and intermolecular crosslinking membrane and cytosol constituents with drastically alteration of membrane viscoelastic and cytosol 
viscous properties with reduction in cell deformability and aggregation. Direct measurement of RBC aggregation has not been frequently used in routine clinical laboratories. The RBC aggregation characteristics can be analyzed using various methods and techniques which are described in the literature (7). Briefly, measurement of erythrocyte sedimentation rate (ESR) (8), lowshear viscometry (9), image analysis (10), dielectric analysis (11), ultrasound backscattering (12) electrical properties $(13,14)$ and photometry (15) are among the methods which have been employed to quantify aggregation.

There are some reports assert that measuring the electrical properties of erythrocyte suspensions can be used for evaluation of erythrocyte aggregation $(7,14,16,17)$. It was reported that electrical properties of erythrocyte suspensions showed a similar trend as photometric measurement and the parameters associated with aggregations could be calculated by using these records $(13,16,18)$. In our previous study, we recorded the photometric and electrical properties of suspensions simultaneously during the erythrocyte aggregation through a glass capillary tube with a diameter of $800 \mu \mathrm{m}$. Recorded light transmittance-time curves (LT) showed a great similarity to the typical curves reflecting erythrocyte aggregation process $(7,15,19,20)$.

This study was designed to compare the time course of electrical impedance and capacitance during $\mathrm{RBC}$ aggregation in a horizontal glass tube. RBC suspended in non-aggregating and aggregating media were investigated; comparisons between measured modalities were based on calculated aggregation indexes that are accepted to reflect RBC aggregation (16) and are used by commercial instruments (15). In this study, it is aimed to determine whether the impedance measurements of erythrocyte suspensions can be used with a different calculation method in determining the degree of erythrocyte aggregation.

\section{Methods}

\section{Blood Samples and Preparation of Red Blood Cell Suspensions}

Venous blood samples were obtained from 10 healthy male volunteers, aged between 20 to 25 years, following the guidelines for hemorheological laboratory methods (16). The study was approved by the Clinical Research Ethical Committee of Bezmialem Vakıf University (19.09.2018). Informed consent was obtained from all participants, and study was conducted according to the Declaration of Helsinki Principles. In addition, adult male Wistar albino rats weighing between 250-300 g were used in this study. Blood samples were taken from abdominal aorta under ketamine-xylazine anesthesia from ten animals into sodium heparin $(15 \mathrm{IU} / \mathrm{mL})$. All animal use procedures were approved by the Laboratory Animals Ethics Committee, Bezmialem Vakıf University. Blood was centrifuged at $1400 \mathrm{~g}$ for $5 \mathrm{~min}$, and plasma was separated carefully and saved. RBC were washed twice with isotonic phosphate-buffered saline (PBS, $\mathrm{pH}=7.4$ ). Samples were immediately evaluated, and studies on samples were completed within four hours following the blood drawing.

\section{Modification of Red Blood Cell Aggregation: Red Blood Cell Suspended in Diluted Plasma}

Plasma was diluted with PBS at ratios of $1 / 2$ corresponding to $50 \%$ of the plasma components in undiluted plasma. Washed $\mathrm{RBC}$ from each donor were re-suspended either in autologous plasma (control) or in diluted plasma at $0.4 \mathrm{l} / \mathrm{l}$ hematocrit.

\section{Modification of Red Blood Cell Aggregation: Red Blood Cell Suspended in $1 \%$ Dextran 500}

Washed RBC from each donor were suspended in plasma containing 1\% Dextran $500(500 \mathrm{kDa}$, Sigma Chemical Company, St. Louis, MO, USA) at $0.41 / 1$ hematocrit.

\section{Modification of Red Blood Cell Aggregation: Glutaraldehyde Treatment of Red Blood Cell}

Washed RBC from each donor were re-suspended in PBS at 0.05 1/1 hematocrit, following which glutaraldehyde (GA, Merck, Darmstadt, Germany) was added to achieve a final concentration of $0.003 \%$ and the suspension incubated at room temperature $\left(22{ }^{\circ} \mathrm{C}\right)$ for $30 \mathrm{~min}$. RBC were then washed three times with PBS after the incubation and re-suspended in autologous plasma at $0.4 \mathrm{l} / 1$ hematocrit.

\section{Measurement System and Experimental Protocol}

RBC aggregation in glass capillaries with internal diameters of $800 \mu \mathrm{m}$ was assessed by monitoring electrical impedance during aggregation process. The measurement system is outlined in Figure 1. Electrical impedance of RBC suspensions were measured in horizontally-alligned, $75 \mathrm{~mm}$ long capillaries, across the two stainless steel electrodes attached to both ends of the capillary. The measurement chamber (glass capillary and electrodes) were placed in a temperature-controlled box and maintained at $37^{\circ} \mathrm{C}$. A computer controlled inductancecapacitance-resistance (LCR)-meter (Hioki, 3532 LCR HiTester, Nagano, Japan) was used for continuous monitoring of electrical impedance parameters between these electrodes, during the RBC aggregation process. The instrument was operated in series mode and a two-electrode configuration was applied. Briefly, the LCR meter applies a test signal across the electrodes at $100 \mathrm{kHz}$ and 1 volt constant voltage $(\mathrm{V})$, measures the resulting current (I) and phase angle $(\theta)$ between $\mathrm{V}$ and $\mathrm{I}$.

RBC suspensions were pumped through the capillary with a syringe pump (World Precision Instruments, Aladdin-4000, Florida, USA) at a calculated flow rate to generate a wall shear rate of $-500 s-1$, for $5 s$, to obtain complete disaggregation, at the start of each measurement procedure. Electrical impedance parameters were recorded at $1 \mathrm{~s}$ intervals for the $120 \mathrm{~s}$ following the stoppage of flow in the capillary tube, via a RS-232 interface on a digital computer.

\section{Calculations and Statistics}

It is known that when erythrocyte aggregation is examined by its property of light reflection or transmittance, the course of time can be modelled by a curve with double-exponential Time courses of $\mathrm{C}$ and $\mathrm{Z}$ data recorded during the aggregation process 
after abruptly stopping flow were used to calculate aggregation index (AI) which is equivalent to those reported by commerciallyavailable RBC aggregometers (Figure 2). Calculations were conducted using a special software written in LabView 8.6 (National Instruments, Austin, Texas, USA). The results of the measurements on RBC suspensions with normal and modified aggregation are expressed as mean \pm standard error. Statistical comparisons were done using one-way analysis of variance followed with Bonferroni's multiple comparison test.

\section{Results}

\section{Time Course of C and Z During Red Blood Cell Aggregation}

Figure 3 a demonstrates the time course $C$, recorded using the same RBC suspensions prepared in 1/2 diluted plasma, dextran and whole plasma. There is a sharp decrease in $\mathrm{C}$ following the stoppage of flow, reaching to a minimum in a few second. This valley is followed by increased $\mathrm{C}$ with a characteristic time course. The initial phase of decreased $\mathrm{C}$ reflects the recovery of RBC morphology for cells initially deformed by shear forces during high-shear flow (corresponding to $-500 \mathrm{~s}-1$ shear rate), with the shape changing from an elongated form to the normal biconcave-discoid shape. The later phase of gradual increases of $\mathrm{C}$ reflects $\mathrm{RBC}$ aggregate formation which follows a characteristic time course.

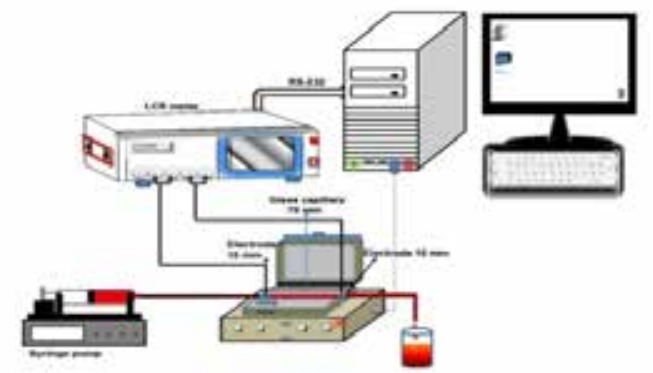

Figure 1. Measurement system LCR: Inductance-capacitance-resistance

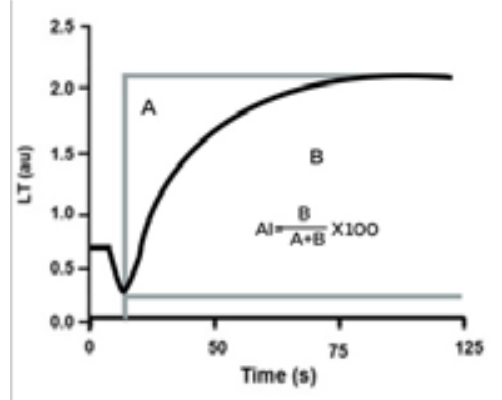

Figure 2. Calculation procedure for aggregation parameters, using light transmittance -time data. " $A$ " and " $B$ " are the areas of the surfaces above and below the curve, respectively. Aggregation index is equal to $B /(A+B)$

Al: Aggregation index, LT: Light transmittance
Capacitance recorded in RBC suspension in dextran, whole blood and 1/2 diluted plasma exhibited similar pattern, although the time course was significantly slower in $1 / 2$ diluted plasma. In capacitance measurements, the changes of plasma samples diluted by PBS after the flow stopped showed a little peak than the plasma samples not diluted by PBS (Figure 3a). There are significant changes in the capacitance measurements in all samples.

The time course of the change in $\mathrm{Z}$ after reaching to the peak values were similar to each other for RBC suspensions in PBS, diluted and undiluted plasma (Figure 3b). A finding of this study was the time course of $\mathrm{Z}$ changes for non-aggregating suspensions of RBC in PBS. $Z$ changes had the same pattern as those for aggregating suspensions, while such similarity between aggregating and non-aggregating suspensions was not observed for C.

\section{Aggregation Characteristics of Red Blood Cell Suspensions}

RBC aggregation parameters calculated using syllectogram recorded from different $\mathrm{RBC}$ suspensions were compared by analyzing data obtained using samples with whole blood, decreased (i.e., $1 / 2$ diluted plasma), and enhanced (i.e., 1\% Dextran 500) aggregation. Aggregation parameters for these samples, as measured by a widely used commercial erythrocyte aggregometer [i.e., Laser-assisted optical rotational cell analyzer (LORCA)], indicated the expected differences (Table 1) $(14,18)$.
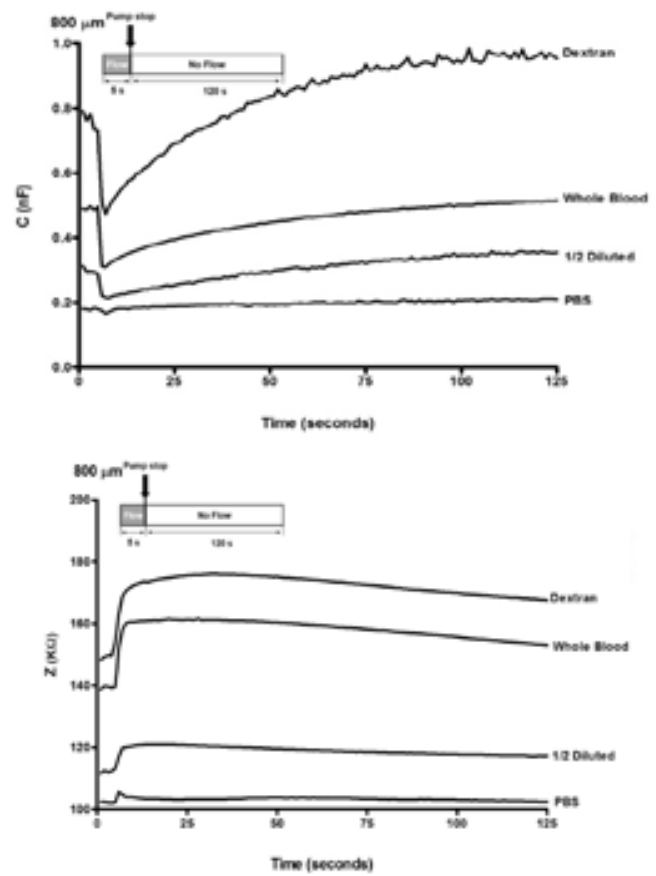

Figure 3. (a) Time course of series capacitance (C) and (b) Impedance ( $Z$ ) across the glass capillary after a sudden stop of flow of red blood cell suspended in dextran, whole blood and 1/2 diluted plasma. Capacitance and impedance were monitored at $100 \mathrm{KHz}$ frequency

PBS: Phosphate-buffered saline 
The AI parameter was significantly decreased for RBC suspended in $1 / 2$ diluted plasma and was increased for RBC suspended in the $1 \%$ dextran 500 solution. It is obvious that AI calculated using $\mathrm{C}$ data exhibited a similar trend for the diluted plasma and plasma with $1 \%$ dextran 500 , with a rank order of plasma + D500 > undiluted plasma >1/2 diluted plasma. This trend was not seen when using $Z$ data.

\section{Electrical Characteristics of Red Blood Cell -plasma Suspensions and Red Blood Cell Aggregation Indexes Calculated Using Z Time Course}

In Figure 4, it is seen that the impedance values of the blood samples prepared in the second minute after stopping the flow are increased. Impedance values in PBS and 40\% hematocrit adjusted samples in plasma showed statistically significant increases in both PBS and plasma samples without erythrocytes $(\mathrm{p}<0.001)$. An interesting result is that although the hematocrit ratios are the same, the impedance value of erythrocyte suspension prepared in plasma is significantly different from the PBS sample $(\mathrm{p}<0.001)$. However, there was no significant difference between PBS and plasma in which there was no cell $(\mathrm{p}>0.05)$.

In Figure 5, the impedance values of the erythrocyte suspension in the plasma are increased due to the increase of the hematocrit value. Gradually increasing the hematocrit values from $20 \%$ to $40 \%$ to $60 \%$ by addition erythrocyte into the plasma significantly increases the impedance values $(\mathrm{p}<0.001)$. As shown in Figure
4 and Figure 5, there is a significant difference between the impedance values of erythrocyte suspensions in PBS and plasma with the same hematocrit value $(40 \%)$. This shows us that the value of the impedance changes according to both the properties of the suspension environment and the number of cells it contains. The impedance values of samples with $40 \%$ hematocrit prepared in PBS and the impedance values of samples with $20 \%$ hematocrit in plasma shown in Figure 5 are very close to each other. This may also be related to the behavior of erythrocytes, besides the number of cells and suspension medium. It is known that erythrocyte aggregation does not occur in simple salt solutions in which there are no macromolecules. Higher values of impedance in samples prepared in plasma may be associated with erythrocyte aggregation. The rise in the impedance value with the increase in hematocrit also explains this case.

In simple salt solutions (eg PBS and $0.9 \% \mathrm{NaCl}$ ) in which no macromolecule is present, rouleaux formation does not occur. As shown in Figure 6, the impedance values of PBS and 0.9\% $\mathrm{NaCl}$ are very close to each other. Erythrocytes in suspension are responsible for the increased impedance in $40 \%$ erythrocyte suspension prepared in PBS. When the PBS and/or $0.9 \% \mathrm{NaCl}$ values are compared with the plasma, the impedance value of the plasma is higher. When the PBS and/or $0.9 \% \mathrm{NaCl}$ values are compared with the plasma, the impedance value of the plasma is higher. Although this difference is not significant $(\mathrm{p}>0.05)$, it is thought that the macromolecules in the plasma may be

Table 1. Aggregation Index values by the commercial erythrocyte aggregometer (i.e., Laser-assisted optical rotational cell analyzer, Myrenne Aggregometer) $(14,18)$ Impedance and Capacitance for red blood cell suspensions prepared in autologous plasma, $1 / 2$ diluted autologous plasma with phosphate-buffered saline corresponding to the $50 \%$ of plasma components and autologous plasma with $1 \%$ dextran 500 in. Data are mean \pm standard error $(n=10)$

Aggregation index (Al)

(au)

Whole blood

$1 / 2$ diluted

1\% 500 kDa Dextran

PBS
LORCA

(Al)

$70.3 \pm 2.3$

$32.6 \pm 2.4^{* * *}$

$82.1 \pm 1.5^{*}$

ND
Myrenne

(M index)

$19.9 \pm 1.1$

$2.5 \pm 1.0$ **

$33.2 \pm 1.6^{* *}$

ND

Capacitance
(C)
$79.0 \pm 1.7$
$60.6 \pm 5.1$ **
$84.4 \pm 1.8$ *
ND

Capacitance

(C)

$60.6 \pm 5.1$ **

ND
Impedance

(Z)

\section{$73.8 \pm 2.5$}

$59.6 \pm 1.5^{* *}$

$69.8 \pm 5.3$

$34.5 \pm 2.3 * *$

Difference from "whole blood"; *: $p<0.05$; **: $p<0.01$, ***: $p<0.001$. au: arbitrary unit ND: Not determined, Al: Aggregation index, PBS: Phosphate-buffered saline, LORCA: Laser-assisted optical rotational cell analyzer

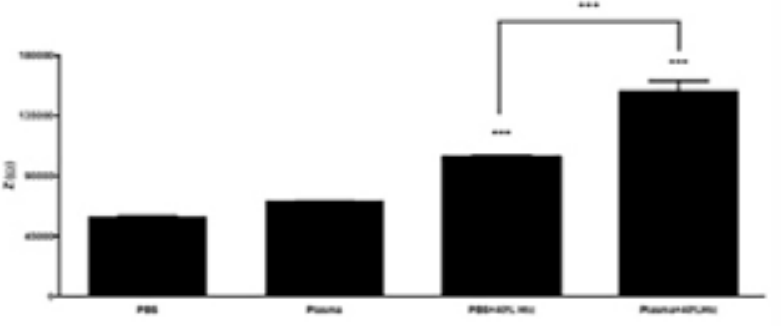

Figure 4. Impedance values of phosphate-buffered saline and plasma samples adjusted to $40 \%$ hematocrit. Data are mean \pm standard error $(n=10)$. Difference from "phosphatebuffered saline and plasma"; ***: $p<0.001,+++$ : $p<0.001$ PBS: Phosphate-buffered saline

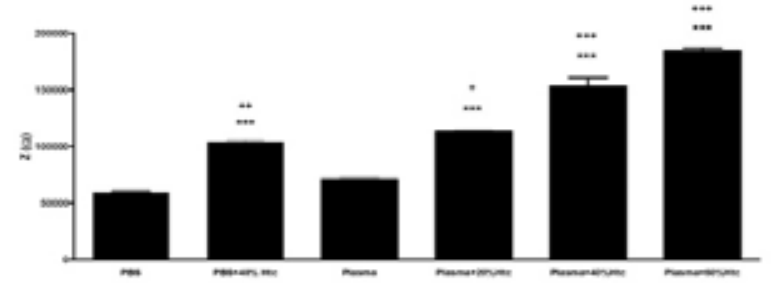

Figure 5. Effect of hematocrit ratio on impedance value. Data are mean \pm standard error $(n=10)$. Difference from "phosphate-buffered saline", ***: $p<0.001$. Difference from "Plasma", +: $p<0.05,+++$ : $<<0.01,+++:$ p $<0.001$

PBS: Phosphate-buffered saline 
responsible for this increment (eg, fibrinogen, etc.). It is assumed that this significant difference $(\mathrm{p}<0.001)$ between the two samples that only the suspension medium is different with the same hematocrit represents erythrocyte aggregation.

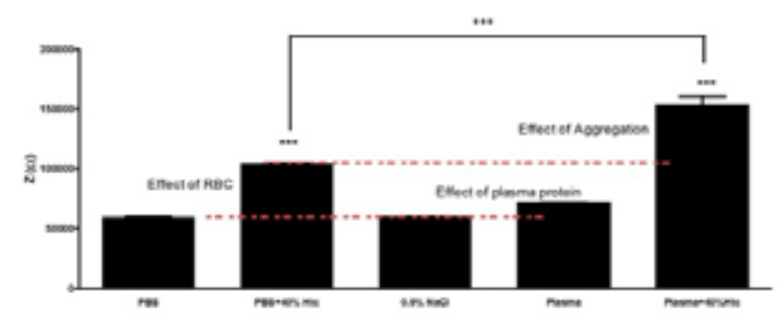

Figure 6. Comparison of phosphate-buffered saline and plasma impedance values. Data are mean \pm standard error $(n=10)$. Difference from "phosphate-buffered saline, $0.9 \%$ $\mathrm{NaCl}$ and plasma", ***: $\mathrm{p}<0.001,+++$ : $\mathrm{p}<0.001$

PBS: Phosphate-buffered saline, RBC: Red blood cell
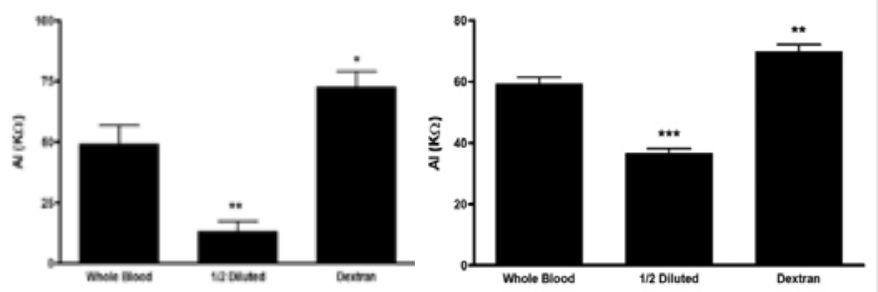

Figure 7. Comparison of calculated impedance values for (a) Human and (b) Rat erythrocyte suspensions. Data are mean \pm standard error $(n=10)$. Difference from "whole blood"; *: $p<0.05$; **: $p<0.01, * * *$ : $p<0.001$

Al: Aggregation index
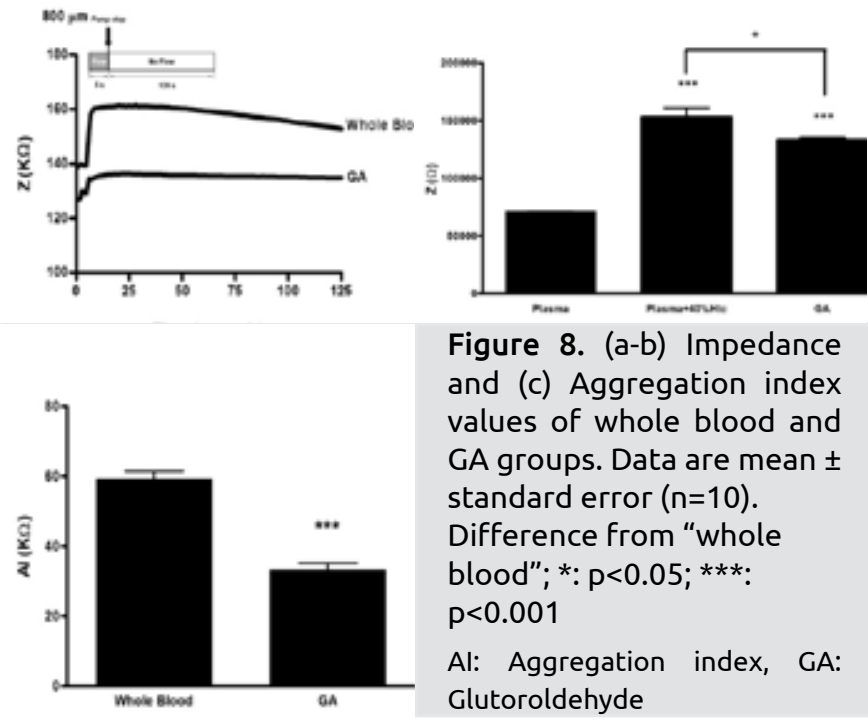

Figure 8. (a-b) Impedance and (c) Aggregation index values of whole blood and GA groups. Data are mean \pm standard error $(n=10)$. Difference from "whole blood"; *: p<0.05; ***: $\mathrm{p}<0.001$

Al: Aggregation index, GA: Glutoroldehyde
Figure 7 shows that when it presented in terms of kilo ohm there is a significant difference $(\mathrm{p}<0.001)$ between the impedance value of the erythrocyte suspension in the hematocrit adjusted to $40 \%$ and the impedance value of the erythrocyte suspension in the plasma with the hematocrit of $40 \%$. Figure 7 a shows that the value of dilution prepared with human blood decreased significantly $(\mathrm{p}<0.01)$ compared to the whole blood group and increased in the dextran group $(\mathrm{p}<0.05)$. When the same study was repeated with rat blood (Figure $7 \mathrm{~b}$ ), the same changes were observed. In dilution and dextran samples where aggregation levels have been modified, a similar trend is seen with photometric devices commonly used for measuring erythrocyte aggregation (Dextran $>$ Whole Blood $>$ Dilution).

Figures $8 \mathrm{a}$ and $8 \mathrm{~b}$ show impedance values for whole blood and GA groups. The impedance value of the erythrocytes treated with GA and suspension prepared with $40 \%$ hematocrit with its own plasma was found to be lower than the whole blood impedance value $(p<0.05)$. These results show that in erythrocytes prepared with the same plasma with the same hematocrit ratio, only the GA-induced structural change resulted in reduced measured impedance values. Similarly, the calculated AI value was significantly reduced compared to the whole blood group (Figure $8 \mathrm{c}, \mathrm{p}<0.001)$.

\section{Discussion}

Aggregation denotes the formation of reversible clumps of RBC under sufficiently low shear stresses $(21,22)$. It has been reported that electrical properties of red cell suspensions also change during aggregation $(11,13,14,23-27)$. The electrical properties of blood plasma and blood cells differ from each other. The plasma and cell interior consist of conducting fluids with certain electrical resistivities and can be simulated by resistors, whereas the cell membranes consist of phospholipids and proteins with dielectric properties and can be simulated by capacitors. Hence, the electrical impedance of blood is primarily characterized by three parameters: plasma resistance $\mathrm{Rp}$, cell interior resistance $\mathrm{R}$; and cell membrane capacitance C (28).

Electrical properties of erythrocyte suspensions are determined by various factors. They include the suspension characteristics (suspension medium, hematocrit and RBC properties), the hydrodynamic conditions (shear forces), the measurement conditions (geometry of the measuring system, the electrodes and measuring the frequency characteristics). Prior studies have suggested that electrical properties of the suspensions may reflect the kinetics of $\mathrm{RBC}$ aggregation if monitored during the aggregation process.

In our previous studies, $\mathrm{C}$ recordings were also obtained using $\mathrm{RBC}$ suspended in isotonic PBS, a suspending medium which did not contain proteins or macromolecules. RBC do not aggregate if they are suspended in such media, and this lack of aggregation was confirmed by "zero" aggregation indexes provided by the photometric aggregometer. The time course of $\mathrm{C}$ after the sudden stop exhibited a different pattern than $Z$. There was no detectable change of $\mathrm{C}$ for the nonaggregating $\mathrm{RBC}$ suspension in PBS. An expected finding of this study was the time course of $\mathrm{Z}$ changes 
for non-aggregating suspensions of RBC in PBS (Table 1). Z changes had the same pattern as those for aggregating suspensions, while such similarity between aggregating and nonaggregating suspensions was not observed for C.

Impedance values in PBS and 40\% hematocrit adjusted samples in plasma showed statistically significant increases in both PBS and plasma samples without erythrocytes (Figure 4). An interesting result is that although the hematocrit ratios are the same, the impedance value of erythrocyte suspension prepared in plasma is significantly different from the PBS sample $(\mathrm{p}<0.001)$. However, there was no significant difference between PBS and plasma in which there was no cell $(\mathrm{p}>0.05)$.

It was reported that measuring the electrical properties of erythrocyte suspensions can be one of the important methods which evaluate the erythrocyte aggregation $(11,25-27)$. In these reports, the electrical properties of plasma and erythrocyte suspensions were evaluated by impedance and dielectric spectroscopy $(11,19,26,29)$. Zhao and Jacopson (29) showed that the values of electrical impedance and capacitance were increased by fibrinogen concentration in suspensions. They also report that ESR was correlated with capacitance and capacitance was very sensitive to erythrocyte sedimentation rate. Pribush et al. $(11,26,27)$ demonstrated that electrical properties of erythrocyte suspensions changed during aggregation. They speculated that monitoring of the electrical properties of these suspensions could give information about the process of aggregations. Capacitance values measured after the sudden cessation of blood flow in the system has changed and this change caused by the RBC aggregation were reported in studies using whole blood and erythrocyte suspensions (11). Electrical properties of erythrocyte suspensions showed a similar trend as photometric measurement and the parameters associated with aggregations could be calculated by using these records were reported $(13,14,25)$.

The LORCA records reflected light (LR) from RBC suspensions while the Myrenne aggregometer record transmitted light (LT). The three types of RBC suspensions (i.e., suspensions in $1 / 2$ diluted plasma, whole blood and plasma containing 1\% dextran 500) exhibited different aggregation characteristics, judged by LORCA and Myrenne aggregometers (Table 1). RBC suspended in $1 / 2$ diluted plasma ( $50 \%$ of original plasma components) represent decreased aggregation, while RBC suspended in $1 \%$ dextran 500 represent markedly increased aggregation; both changes of aggregation are due to alterations of suspending phase composition. The decreased concentrations of plasma components due to dilution resulted in significant and progressive changes of AI as measured by the three methods (LT, LR and C).

In our previous studies $(13,17)$, we recorded the photometric and electrical properties of suspensions simultaneously during the erythrocyte aggregation of glass capillary tubes with a diameter of $1000 \mu \mathrm{m}$. We evaluated the correlations of AI of erythrocyte suspensions measured by photometric aggregometer and AI obtained from calculating the area under curve of light transmission, impedance and capacitance measurements and found that there was a positive and statistically significant correlation between capacitance value/light transmission and AI.
There was no significantly correlation between impedance and AI value. According to results of this pre study, it was suggested that capacitance values monitored during erythrocyte aggregation in capillary tubes were reflected the aggregation time period. This suggestion was stated by comparisons using simultaneously records of LT.

When the PBS and/or $0.9 \% \mathrm{NaCl}$ values are compared with the plasma, the impedance value of the plasma is higher. Although this difference is not significant $(\mathrm{p}>0.05)$, it is thought that the macromolecules in the plasma may be responsible for this increment (eg, fibrinogen, etc.). It is assumed that this significant difference $(p<0.001)$ between the two samples that only the suspension medium is different with the same hematocrit represents erythrocyte aggregation (Figure 6 and 7). Only, the GA-induced structural change resulted in reduced measured impedance values in erythrocytes prepared with the same plasma with the same hematocrit ratio Similarly, the calculated AI value was significantly reduced compared to the whole blood group (Figure 8) It was obvious from all these results, the impedance value of the erythrocyte suspensions is influenced by the composition of the suspension medium, the number of erythrocytes in suspension (hematocrit) and the structural properties of erythrocytes.

\section{Conclusions}

We aimed in this study to determine whether the impedance measurements of erythrocyte suspensions can be used with a different calculation method in determining the degree of erythrocyte aggregation. Although impedance measurements of erythrocyte suspensions do not allow for the calculation of erythrocyte aggregation kinetics ( $\mathrm{T}$ fast and $\mathrm{t} 1 / 2$ ), it is thought that they can be used as "AI" which indicates the degree of erythrocyte aggregation. In this way, AI can be measured in a simpler and repeatable way.

\section{Ethics}

Ethics Committee Approval: The study was approved by the Clinical Research Ethics Committee of Bezmialem Vakıf University (19.09.2018).

Informed Consent: Informed consent was obtained from all participants, and study was conducted according to the Declaration of Helsinki Principles.

Peer-review: Externally peer-reviewed.

\section{References}

1. Baskurt OK. In vivo correlates of altered blood rheology. Biorheology 2008;45:629-38.

2. Meiselman HJ. Red blood cell aggregation: 45 years being curious. Biorheology 2009;46:1-19.

3. Rampling MW, Meiselman HJ, Neu B, Baskurt OK. Influence of cell-specific factors on red blood cell aggregation. Biorheology 2004;41:91-112.

4. Rampling MW. Haemorheology and the inflammatory process. Clin Hemorheol Microcirc 1998;19:129-32. 
5. Baskurt OK, Temiz A, Meiselman HJ. Red blood cell aggregation in experimental sepsis. J Lab Clin Med 1997;130:183-90.

6. O.K.Baskurt, M.R. Hardeman, M.W. Rampling and H.J. Meiselman. In vivo hemorheology iHoHaH, IOS Press, Amsterdam 2007;322-38.

7. Baskurt OK, Meiselman HJ, Kayar E. Measurement of red blood cell aggregation in a "plate-plate“ shearing system by analysis of light transmission. Clin Hemorheol Microcirc 1998;19:307-14.

8. Houbouyan LL, Delamaire M, Beauchet A, Gentil M, Cauchois G, Taccoen A, et al. Multicenter study of an erythro-aggregometer: quality control and standardization. Clin Hemorheol Microcirc 1997;17:299-306.

9. Baskurt OK, Meiselman HJ. Cellular determinants of low-shear blood viscosity. Biorheology 1997;34:235-47.

10. Berliner S, Zeltser D, Shapira I, Assayag EB, Mardi T, Serov J, et al. A simple biomarker to exclude the presence of low grade inflammation in apparently healthy individuals. J Cardiovasc Risk 2002;9:281-6.

11. Pribush A, Meiselman HJ, Meyerstein D, Meyerstein N. Dielectric approach to the investigation of erythrocyte aggregation: I. Experimental basis of the method. Biorheology 1999;36:411-23.

12. Rouffiac V, Peronneau P, Hadengue A, Barbet A, Delouche P, Dantan $P$, et al. A new ultrasound principle for characterizing erythrocyte aggregation: in vitro reproducibility and validation. Invest Radiol 2002;37:413-20.

13. Baskurt OK, Uyuklu M, Meiselman HJ. Simultaneous monitoring of electrical conductance and light transmittance during red blood cell aggregation. Biorheology 2009;46:239-49.

14. Baskurt OK, Uyuklu M, Meiselman HJ. Time course of electrical impedance during red blood cell aggregation in a glass tube: comparison with light transmittance. IEEE Trans Biomed Eng 2010;57:969-78.

15. Hardeman MR, Dobbe JG, Ince C. The Laser-assisted Optical Rotational Cell Analyzer (LORCA) as red blood cell aggregometer. Clin Hemorheol Microcirc 2001;25:1-11.

16. Baskurt OK, Boynard M, Cokelet GC, Connes P, Cooke BM, Forconi $\mathrm{S}$, et al. New guidelines for hemorheological laboratory techniques. Clin Hemorheol Microcirc 2009;42:75-97.

17. Baskurt OK, Uyuklu M, Hardeman MR, Meiselman HJ. Photometric measurements of red blood cell aggregation: light transmission versus light reflectance. J Biomed Opt 2009; 14:540-44.
18. Baskurt OK, Uyuklu M, Ulker P, Cengiz M, Nemeth N, Alexy T, et al. Comparison of three instruments for measuring red blood cell aggregation. Clin Hemorheol Microcirc 2009;43:283-98.

19. Gaw RL, Cornish BH, Thomas BJ. The electrical impedance of pulsatile blood flowing through rigid tubes: a theoretical investigation. IEEE Trans Biomed Eng 2008;55:721-7.

20. Kiesewetter H, Radtke H, Schneider R, Mussler K, Scheffler A, Schmid-Schonbein $\mathrm{H}$. [The mini-erythrocyte aggregometer: a new apparatus for the rapid quantification of the extent of erythrocyte aggregation]. Biomed Tech (Berl) 1982;27:209-13.

21. M.W. Rampling. Red cell aggregation and yield stress. In: Clinical blood rheology; Lowe GDO ECP, Inc Florida 1988;45-64.

22. H.J. Meiselman. Red blood cell role in RBC aggregation: 1963-1993 and beyond. Clin Hemorheol Microcirc 1993;13:575-92.

23. Antonova N, Riha P, Ivanov I. Time dependent variation of human blood conductivity as a method for an estimation of RBC aggregation. Clin Hemorheol Microcirc 2008;39:69-78

24. Balan C, Balut C, Gheorghe L, Gheorghe C, Gheorghiu E, Ursu G. Experimental determination of blood permittivity and conductivity in simple shear flow. Clin Hemorheol Microcirc 2004;30:359-64.

25. Pribush A, Hatzkelson L, Meyerstein D, Meyerstein N. A novel technique for quantification of erythrocyte aggregation abnormalities in pathophysiological situations. Clin Hemorheol Microcirc 2007;36:121-32.

26. Pribush A, Meiselman HJ, Meyerstein D, Meyerstein N. Dielectric approach to investigation of erythrocyte aggregation. II. Kinetics of erythrocyte aggregation-disaggregation in quiescent and flowing blood. Biorheology 2000;37:429-41.

27. Pribush A, Meyerstein D, Meyerstein N. Conductometric study of shear-dependent processes in red cell suspensions. I. Effect of red blood cell aggregate morphology on blood conductance. Biorheology 2004;41:13-28.

28. Fricke H, Morse S. The Electric Resistance and Capacity of Blood for Frequencies between 800 and 4(1/2) Million Cycles. J Gen Physiol 1925;9:153-67.

29. Zhao TX, Jacobson B. Quantitative correlations among fibrinogen concentration, sedimentation rate and electrical impedance of blood. Med Biol Eng Comput 1997;35:181-5. 